\title{
Inventario de supresión del pensamiento del "oso blanco" (WBSI): Propiedades psicométricas de la versión portuguesa (Portugal)
}

\author{
Antonia M. Jiménez $\operatorname{Ros}^{1}$, Alejandro Orgambidez $\operatorname{Ramos}^{1}$ y Luis M. Pascual Orts ${ }^{2}$ \\ ${ }^{1}$ Universidad del Algarve, Faro, Portugal \\ ${ }^{2}$ Universidad de Zaragoza, Zaragoza, España
}

Resumen: El White Bear Suppression Inventory (WBSI) fue concebido para evaluar tendencia a la supresión de pensamientos. En el presente estudio se aplicó, junto con otras medidas, a una muestra de 558 sujetos de la población no clínica portuguesa para el estudio de su estructura factorial y propiedades psicométricas. Se sometieron a análisis factorial confirmatorio (AFC) cinco modelos con estructuras unidimensionales, bidimensionales y tridimensionales. Los resultados mostraron un factor con una adecuada consistencia interna y estabilidad temporal $\alpha=.88, \mathrm{CCI}=.61$ [IC95\% $=.31-.78]$ ). La escala obtenida mostró asociaciones con medidas psicológicas y psicopatológicas presentando una adecuada validez convergente. Se presentan los datos normativos para la muestra analizada. Se concluye que la versión portuguesa del WBSI se presenta como un instrumento adecuado para su utilización en Portugal.

Palabras clave: WBSI; supresión de pensamiento; análisis factorial exploratorio y confirmatorio; fiabilidad; validez.

The White Bear Suppression Inventory (WBSI): Psychometric properties of the Portuguese (Portugal) version

\begin{abstract}
The White Bear Suppression Inventory (WBSI) was developed to measure the tendency to suppress unwanted negative thought. With the aim to study its internal structure and psychometrics properties, this inventory was applied with other measures to a sample of 558 participants from the general non-clinical Portuguese population. We performed confirmatory factorial analyses (AFC) to test five models of unidimensional, bi-dimensional and three-dimensional structures. The results yielded the existence of one single factor with adequate levels of internal consistency and temporal stability $(\alpha=.88$, CCI $=.61$ $[$ IC95\% = .31-.78]). The WBSI was associated with psychological and psychopathological measures showing adequate levels of convergent validity. We present the normative data for the analyzed sample. In conclusion, the Portuguese version of the WBSI is an adequate instrument to be used in Portugal.
\end{abstract}

Keywords: WBSI, thought suppression; exploratory and confirmatory factorial analysis; reliability; validity.

\section{Introducción}

Los intentos deliberados de no pensar en un pensamiento particular parecen conducir a un incremento paradójico en la frecuencia y malestar causado por tal pensamiento. Este efecto paradójico ocurre bien inmediatamente, bien cuando cesan los esfuerzos de supresión. Estos fueron los resultados fundamentales de la investigación conducida por Wegner, Schneider, Carter y White (1987). Desde entonces, la supresión de pensamiento ha sido y continúa siendo un fenómeno ampliamente estudiado. Se ha estudiado en el ámbito del trastorno obsesivo-compulsivo (TOC) pero también en muchas otras patologías tales como la fobia social (Muris, Merckelbach, Horselenberg, Sijsenaar y Leeuw, 1997) o la depresión (Wenzlaff, Wegner y Roper, 1988).
Recibido: 16 julio 2014; aceptado: 10 diciembre 2014.

Correspondencia: Antonia María Jiménez Ros, Departamento de Psicologia e Ciências da Educação, Faculdade de Ciências Humanas e Sociais, Campus de Gambelas, 8005-139 Faro, Portugal. Correo-e: aros@ualg.pt
Agradecimientos: Nuestro sincero agradecimiento al profesor Daniel Wegner de la Universidad de Harward por la autorización para la utilización de este inventario. Agradecemos así mismo a los revisores por los valiosos comentarios que nos han servido para mejorar el manuscrito original. 
Las personas varían en la tendencia natural para suprimir pensamientos indeseados (Wenzlaff y Wegner, 2000). Por este motivo, Wegner y Zanakos (1994) consideraron que si fuese posible aislar individuos que regularmente suprimen sus pensamientos, se podrían también encontrar otras características que los hacen más vulnerables al desarrollo de psicopatología. De forma más específica, consideraron que la supresión crónica debería estar asociada con la ocurrencia de pensamientos obsesivos y que debería también existir una relación positiva entre los intentos crónicos de supresión y los niveles de ansiedad y depresión. Con el objetivo de evaluar los intentos crónicos de supresión, estos autores desarrollaron una medida de auto-informe, el White Bear Supression Inventory (WBSI). Partieron de 72 ítems que, tras la aplicación a varias muestras de tamaño considerable y sometido a sucesivos análisis factoriales exploratorios, quedaron reducidos a 15. El instrumento mostró una adecuada estabilidad temporal y se asoció de forma positiva con pensamientos obsesivos, depresión, ansiedad estado y sensibilidad a la ansiedad.

Los autores concluyeron que se trataba de una medida fiable que podía evaluar la supresión como rasgo y que podría permitir, junto con otras medidas, seleccionar los sujetos más vulnerables al desarrollo de psicopatología.

Algunas de las versiones posteriores de este instrumento han puesto en entredicho la estructura interna del mismo. A favor de la unidimensionalidad, encontramos los resultados de los análisis factoriales exploratorios realizados por Palm y Strong (2007) que replican la estructura encontrada por Wegner y Zanakos (1994), así como los obtenidos por Muris, Merckelbach y Horselenberg (1996) quienes encontraron, tal como los autores del instrumento original, una única dimensión de supresión de pensamiento. Sin embargo, los resultados de este estudio suscitaban dudas sobre algunos ítems que no parecían evaluar supresión. Posteriormente otros autores utilizando también análisis factoriales exploratorios (i.e., Blumberg, 2000; Höping y de Jong-Meyer, 2003; y Rassin, 2003) y confirmatorios (i.e., Blumberg, 2000; Luciano et al., 2006 y González, Avero, Rovella y $\mathrm{Cu}-$ bas, 2008) obtuvieron estructuras bidimensionales o tridimensionales, compuestas por un factor de supresión y otro/s de intrusión y/o de distracción.

En la totalidad de los resultados de las investigaciones consultadas que estudian la estructura interna del WBSI, emerge, de forma consistente, una dimensión de supresión de pensamiento. El número y contenido de los ítems que la integran no es, sin embargo, siempre tan consistente. En algunas investigaciones, la supresión de pensamiento surge como un factor dominante que expli- ca el mayor porcentaje de la varianza y aglutina una gran parte de los ítems (i.e., Rassin, 2003) pero, en otras, surge como una dimensión secundaria (i.e., Blumberg, 2000 y Höping y de Jong-Meyer, 2003). Las dos dimensiones restantes (intrusión y distracción) surgen, de forma todavía menos homogénea en las diferentes versiones del WBSI. Los ítems que se agrupan en cada uno de estos factores tampoco son consistentes, variando en número y contenido. Schmidt y colaboradores (2009) sintetizan la generalidad de las estructuras del WBSI encontradas en los estudios anteriores.

Consideramos que la poca homogeneidad de estos resultados puede ser debida, por lo menos en parte, a los diferentes abordajes metodológicos y técnicas estadísticas utilizadas en los análisis factoriales exploratorios. Mientras que Wegner y Zanakos (1994) optaron en el estudio original del instrumento por el método de extracción de ejes principales, la mayor parte de los autores posteriores optaron por utilizar el análisis de componentes principales como método de extracción (i.e., Höping y de Jong-Meyer, 2003; Muris et al., 1996; Spinhoven y van der Does, 1999; Rassin, 2003), y unos pocos, recurrieron al método de ejes principales a partir de matrices de correlaciones policóricas al encontrar que las respuestas de su muestra a los ítems del instrumento presentan una simetría o curtosis excesiva (i.e., Palm y Strong, 2007).

Las opciones por los métodos de rotación empleados fueron también diferentes. Algunos autores recurrieron a métodos de rotación ortogonal (i.e., Höping y de Jong-Meyer, 2003; Wegner y Zanakos 1994) y otros optaron por la rotación oblicua (i.e., Blumberg, 2000; Rassin, 2003).

La tercera de las diferencias se situó en la elección de los métodos para la determinación del número de factores. Las mayor parte de las investigaciones determinaron el número de factores combinando la inspección visual del grafico de sedimentación y la regla de Kaiser-Guttman (i.e., Blumberg, 2000; Höping y de Jong-Meyer, 2003; Muris et al., 1996; Rassin, 2003; Wegner y Zanakos, 1994). Las más recientes, sin embargo, combinan el análisis paralelo con la observación del gráfico de sedimentación y el test de la correlación parcial mínima (MAP) (i.e., González et al., 2008; Palm y Strong, 2007).

A pesar de la abundante investigación sobre el WBSI, no existe acuerdo sobre la estructura factorial del mismo, consideramos que, tal vez, los métodos estadísticos utilizados en otros estudios puedan haber sobreestimado el número de factores.

A pesar de la falta de consenso en lo que toca a la estructura interna de este instrumento, los resultados de las diferentes versiones del WBSI revelaron una adecua- 
da consistencia interna con valores de $\alpha=.88$ (Höping y de Jong-Meyer, 2003) y $\alpha=.89$ (Muris et al., 1996 y González et al., 2008). La estabilidad temporal obtenida fue asimismo muy satisfactoria, con valores comprendidos entre $\mathrm{r}=.71$ (González et al., 2008 ) y r=.93 (Höping y de Jong-Meyer, 2003). El total del WBSI mostró asociaciones positivas con otras variables psicológicas y psicopatológicas tales como ansiedad y depresión (Muris et al., 1996; Wegner y Zanakos, 1994), así como con neuroticismo, obsesiones, intrusiones, estrategias de control de pensamiento (Muris et al.,1996), evitación emocional y preocupación (Palm y Strong, 2007; Sandín, Chorot, Valiente, y Lostao, 2009).

El objetivo del presente estudio fue proporcionar evidencias de validez factorial y convergente de la versión portuguesa del WBSI (Wegner y Zanakos, 1994). Para ello, se comprobará cuál de las estructuras factoriales observadas en otros estudios, unidimensional (Muris et al.,1996; Palm y Strong, 2007; Wegner y Zanakos, 1994), de dos factores (González et al., 2008; Höping y De Jong-Meyer, 2003; Rassin, 2003) o de tres factores (Blumberg, 2000) se ajusta mejor a los datos. Igualmente, se analizará la fiabilidad de la versión portuguesa del WBSI y se describirán las relaciones entre la supresión de pensamientos (WBSI), obsesiones y compulsiones (MOCI), ansiedad (STAI), preocupación excesiva (PSWQ) y estrategias de control de pensamientos negativos (TCQ), para comprobar la validez convergente.

\section{Método}

\section{Participantes}

La muestra estuvo compuesta por 558 voluntarios pertenecientes a la población general portuguesa. El $65.5 \%$ eran mujeres $(n=371)$. Cincuenta de los 558 participantes cumplimentaron posteriormente el WBSI tras un período de 3 a 6 semanas ( $58 \%$ mujeres; $M_{\text {edad }}=29.18$, $D T=11.93)$ sin observarse diferencias estadísticamente significativas en las variables socio-demográficas: edad $\left(t_{(554)}=1.35, p=.18\right) \mathrm{y} \operatorname{sexo}\left(X_{(1)}^{2}=1.18 ; p=.28\right)$.

\section{Instrumentos}

Inventario de Supresión del "Oso Blanco" (The White Bear Supression Inventory, WBSI; Wegner y Zanakos, 1994). Este cuestionario pretende evaluar los intentos crónicos de supresión de pensamientos. Consta de 15 ítems que se responden en una escala de 5 niveles de respuesta que van desde 1 (completamente en desacuerdo) a 5 (completamente de acuerdo). La puntuación total se obtiene sumando las respuestas de cada ítem, oscilan- do entre 15 y 75. Cuanto mayor es la puntuación total, mayor es la tendencia para suprimir.

Cuestionario sobre el control del Pensamiento (Thought Control Questionnaire, TCQ; Wells y Davies, 1994). Se utilizó la versión portuguesa validada del TCQ (Jiménez-Ros, 2010). Este cuestionario de 30 ítems agrupados en 5 dimensiones - Distracción, Revaloración, Preocupación, Control Social y Castigo - mide las estrategias que los individuos emplean para controlar su pensamiento. Los 30 ítems se responden de acuerdo con 4 niveles de respuesta que van de 1 (nunca utilizo esa estrategia a 4 (la utilizo casi siempre). Mayores puntuaciones indican una mayor tendencia para la utilización de estrategias de control. Los coeficientes alpha de Cronbach registrados en este estudio fueron .83 para Distracción, .75 para Revaloración, .70 para Preocupación, .72 para Control Social y .73 para Castigo.

Cuestionario de Preocupación de Pensilvania (The Penn State Worry Questionnaire, PSWQ; Meyer, Miller, Metzger, y Borkovec, 1990). Se aplicó la versión portuguesa validada de la PSWQ (Jiménez-Ros, 2010). Esta escala está compuesta por 16 ítems y evalúa la tendencia de los individuos a preocuparse de forma excesiva. Las respuestas son dadas en 5 niveles de respuesta que van de 1 (no es nada característico de mi) a 5 (es muy característico de mi). Mayores puntuaciones indican mayores niveles de preocupación excesiva en el sujeto. La fiabilidad de la escala, medida a través del coeficiente alpha de Cronbach, fue de 80 .

Inventario de Obsesiones y Compulsiones de Mausdley (The Mausdley Obsessive-Compulsive Inventory, MOCI; Hodgson y Rachman, 1977). Se utilizó la versión portuguesa y validada de este instrumento (Jiménez-Ros, 2010). Este instrumento, compuesto por 30 ítems con un formato de respuesta verdadero-falso, permite evaluar los diferentes tipos de trastorno obsesivo. Proporciona una puntuación global sobre los síntomas obsesivos compulsivos y cuatro subtotales referentes a los síntomas de verificación, limpieza, lentitud y duda. Mayores puntuaciones indican mayor presencia de síntomas relacionadas con los trastornos obsesivos. La consistencia interna de la escala, calculada a través del coeficiente alpha de Cronbach, registrada en este estudio fue de .70.

Inventario de Ansiedad Estado-Rasgo (The State-Trait Anxiety Inventory, STAI; Spielberger, Gorsuch, Lushene, Vagg y Jacobs, 1983). Se usó la versión portuguesa de Ponciano et al. (2008). Se trata de una medida de auto-informe compuesta por 40 ítems. Los primeros 20 ítems (STAI-E) evalúan la ansiedad-estado referida a cómo se siente el sujeto en el momento actual (condición emocional transitoria), mientras que los siguientes 20 ítems evalúan ansiedad-rasgo (STAI-R), asociada a cómo se sienta 
el sujeto generalmente (propensión ansiosa relativamente estable). Los ítems se responden de acuerdo con 4 niveles de respuesta que van de 1 (nada) a 4 (mucho). Mayores puntuaciones indican mayores niveles de ansiedad, tanto rasgo como estado. El coeficiente de fiabilidad alpha de Cronbach obtenido fue de .92 para la escala de ansiedad-estado, y .88 para la escala de ansiedad-rasgo.

\section{Procedimiento}

En relación a la adaptación al portugués de la escala WBSI, se utilizaron las recomendaciones de Hambleton, Merenda y Spielberger (2006). En un primer momento se realizó la traducción directa de los ítems originales del inglés al portugués para, posteriormente, llevar a cabo la retraducción del portugués al inglés. Este proceso de doble-traducción fue realizado por traductores profesionales y especialistas en la temática, los cuales fueron informados del objetivo de la escala y del formato de respuesta. Finalmente, dos psicólogos especialistas en el área revisaron la versión final realizando modificaciones en los ítems que lo requerían. El resultado final de este proceso fue la versión portuguesa del Inventario de Supresión del Oso Blanco, compuesto por 15 ítems y con la misma escala de respuesta que en la escala original en inglés, es decir, una escala de 5 puntos desde " 1 " (completamente en desacuerdo) hasta " 5 " (completamente de acuerdo). El Anexo 1 recoge la versión en portugués del WBSI.

Una vez traducido el inventario al portugués, se procedió a su aplicación en primer lugar a una muestra de estudiantes de una universidad pública portuguesa, que rellenaron el protocolo de evaluación de forma colectiva y en presencia de los investigadores. Posteriormente se reclutaron y entrenaron varios colaboradores para la aplicación del cuestionario en la población general. De entre los colaboradores, uno de ellos fue seleccionado y entrenado para la aplicación del protocolo a 50 voluntarios de la población general. Estos 50 voluntarios de la población respondieron nuevamente al cuestionario transcurrido un intervalo de tiempo que varió entre tres y seis semanas. En todos los casos, la participación en el estudio fue voluntaria y anónima, de forma que se dio libertad a los participantes de completar o no los datos socio-demográficos, a excepción de la fecha de nacimiento y el sexo. El orden de aplicación de los cuestionarios fue contrabalanceado en seis versiones para contrarrestar los efectos del cansancio.

\section{Análisis de datos}

El análisis de datos se realizó utilizando el paquete estadístico STATA 12. Las propiedades psicométricas de la escala fueron exploradas a través de análisis factoriales exploratorios y confirmatorios, el análisis de la consistencia interna y la validez convergente o de criterio, y se calcularon los datos normativos. Para los análisis factoriales exploratorios y confirmatorios, la muestra fue distribuida aleatoriamente en dos submuestras $\left(n_{1}=279\right.$ participantes y $n_{2}=279$ participantes).

Para el análisis factorial exploratorio, previamente se calcularon los coeficientes Kaiser-Meyer-Olkin (KMO) y el test de esfericidad de Bartlett para comprobar la idoneidad de los datos para el análisis factorial. Se utilizó el método de extracción de ejes principales con rotación Oblimin. Este método es especialmente recomendado para el análisis exploratorio ya que permite explorar la estructura subyacente de una matriz de datos (Osborne y Fitzpatrick, 2011). Se aplicó la rotación oblicua (Oblimin) porque (a) esperamos, de acuerdo con la revisión teórica realizada, que los posibles factores se encuentren relacionados y (b) los resultados entre extracción ortogonal y oblicua son muy parecidos en el caso de no haber relaciones entre factores (Cabrera-Nguyen, 2010). Para determinar el número de factores a extraer se utilizaron los siguientes criterios (Cabrera-Nguyen, 2010; Osborne y Fitzpatrick, 2011): el criterio de Kaiser de autovalores superiores a la unidad, el test de screen y el análisis paralelo.

Se llevaron a cabo varios análisis factoriales confirmatorios mediante el procedimiento de máxima verosimilitud. Este método asume la normalidad de los datos, considerándose robusto cuando este presupuesto no se cumple (Marôco, 2010). Se calcularon los siguientes indicadores conforme a las recomendaciones de Byrne (2001): $\chi^{2}$, la razón entre chi-cuadrado y los grados de libertad $\left(\chi^{2} / g l\right)$ dado que el tamaño de la muestra puede afectar al valor $\chi^{2}$, CFI (comparative fit index), TLI (Tucker-Lewis index), RMSE (root mean square error of approximation) y SRMR (standardized root mean square residual). Cocientes de $\chi^{2}$ entre grados de libertad inferiores a 5 indican un buen ajuste. Por otro lado, se considera aceptable el ajuste del modelo cuando los valores de CFI y TLI son iguales o superiores a .90, y cuando los coeficientes de RMSEA y SRMS son iguales o inferiores a .08 (Hu y Bentler, 1999).

La consistencia interna de la escala se calculó utilizando el coeficiente alpha de Cronbach, así como la estabilidad temporal (fiabilidad test-retest) y la fiabilidad de análisis de ítems (correlación corregida ítem-escala). Se consideraron valores adecuados de alpha de Cronbach los situados en torno a .70 (Nunnally y Berstein, 1995). La fiabilidad test-retest fue evaluada a través del cálculo del coeficiente de correlación intraclase (CCI). Se consideró que valores por debajo de .40 indican baja 
fiabilidad, valores entre .40 y .75 fiabilidad entre regular buena, y valores superiores a .75 como una fiabilidad excelente (Fleiss, 1986).

La validez convergente fue estudiada analizando las correlaciones entre la puntuación en la escala WBSI y las puntuaciones en las escalas TCQ, PSWQ, MOCI y STAI. Se consideraron, de acuerdo con Cohen (1988), correlaciones bajas las comprendidas entre .10 y .29 , medias entre .30 y .49 , y elevadas a partir de .50 .

\section{Resultados}

\section{Análisis factorial exploratorio}

Utilizando la submuestra $n_{1}$ (279 participantes), los ítems del WBSI fueron sometidos a un análisis factorial exploratorio utilizando el método de extracción de ejes principales con rotación oblicua. El test de Kaiser-Meyer-Olkin (KMO) arrojó un valor de .89 y la prueba de esfericidad de Bartlett fue estadísticamente significativa $\left(X_{(105)}^{2}=2477.52, p<.01\right)$, por lo que puede concluirse la idoneidad de los datos para el análisis factorial exploratorio. En el análisis con extracción mediante el análisis de ejes principales y rotación Oblimin, los valores de los primeros cinco autovalores fueron los siguientes: 5.24, $.88, .62, .33$ y .26 . El análisis del criterio de Kaiser (autovalores superiores a la unidad) (Kaiser, 1961), el test de scree (Cattell y Vogelmann, 1977) y el análisis paralelo (O'Connor, 2000) indicaron la existencia de un único factor que explica el $58.9 \%$ de la varianza total observada. La Tabla 1 recoge los datos relativos a la estructura factorial (pesos factoriales y comunalidad) obtenida en el análisis factorial exploratorio. Todos los ítems presentaron saturaciones por encima de .40 .

\section{Análisis factorial confirmatorio}

Con la submuestra $n_{2}$ (279 participantes) se llevaron a cabo varios análisis factoriales confirmatorios con el objetivo de poner a prueba varios modelos teóricos sobre la estructura del WBSI, basados en los resultados obtenidos en nuestro análisis factorial exploratorio y en las estructuras de dos y tres factores observadas en otros estudios. La revisión de la literatura indica que el WBSI podría ajustarse tanto a una estructura unidimensional (Muris et al.,1996; Palm y Strong, 2007; Wegner y Zanakos, 1994) como bidimensional (i.e., González et al., 2008; Höping y de Jong-Meyer, 2003; Rassin, 2003) y tridimensional (i.e., Blumberg, 2000).

El Modelo 1 consiste en una estructura unifactorial conforme a la obtenida en el análisis exploratorio y en los estudios de Palm y Strong, 2007 y Wegner y Zanakos
Tabla 1. Análisis factorial exploratorio del WBSI. Pesos factoriales y comunalidad $\left(h^{2}\right)$ obtenidos a través del método de ejes principales

\begin{tabular}{lcc}
\hline & \multicolumn{2}{c}{ Factor } \\
\cline { 2 - 3 } Ítems WBSI & F1 & $h^{2}$ \\
\hline 1. Hay cosas que prefiero no pensar & .43 & .19 \\
2. A veces me pregunto por qué tengo determinados & .46 & .22 \\
pensamientos & & \\
3. Tengo pensamientos que no puedo parar & .62 & .39 \\
4. Hay imágenes que me vienen a la mente y que no & .63 & .40 \\
puedo borrar & & \\
5. Mis pensamientos frecuentemente giran alrede- & .50 & .25 \\
dor de una sola idea & & \\
6. Desearía dejar de pensar en ciertas cosas & .64 & .42 \\
7. A veces mi mente va tan deprisa que desearía po- & .66 & .44 \\
der pararla & & \\
8. A veces trato de alejar los problemas de mi mente & .46 & .22 \\
9. Hay pensamientos que se me quedan rondando & .66 & .44 \\
por la cabeza & & \\
10. A veces me mantengo ocupado para no dejar que & .57 & .32 \\
mis pensamientos se entrometan en mi mente & & \\
11. Hay cosas sobre las que intento no pensar & .59 & .35 \\
12. A veces desearía poder parar mi pensamiento & .76 & .58 \\
13. A menudo hago cosas para distraerme de mis & .54 & .29 \\
pensamientos & & \\
14. A menudo tengo pensamientos que trato de evitar & .70 & .50 \\
15. Tengo muchos pensamientos que no digo a nadie & .52 & .27 \\
\hline
\end{tabular}

(1994). El Modelo 2 parte de la estructura bifactorial encontrada en la versión alemana de Höping y de Jong-Meyer (2003). El Modelo 3 replicó la estructura bifactorial encontrada en la versión holandesa elaborada por Rassin (2003). El Modelo 4 consiste en la estructura bifactorial obtenida en el estudio de González et al. (2008) sobre la versión española. Finalmente, el Modelo 5 se basó en la estructura trifactorial encontrada por Blumberg (2003). La Tabla 2 muestra la información de los índices de ajuste indicados en el apartado de análisis de datos para todos los Modelos, así como los ítems que componen cada factor.

Los valores de asimetría y curtosis de todos los ítems presentaron valores que no se alejan excesivamente de los considerados como adecuados para asumir el presupuesto de normalidad ( $|\mathrm{Sk}|<3$ y $|\mathrm{Ku}|<10$ ) (Marôco, 2010). El Modelo 4, basado en la estructura observada por González et al. (2008), mostró un mejor ajuste a los datos que el resto de modelos: $\chi^{2}(76)=274.69, p<.01$, $\chi^{2} / g l=3.61, C F I=.84, T L I=.81, R M S E A=.10(\mathrm{IC} 90 \%$ $.088-.113)$ y $S R M R=.061$. No obstante, los coeficientes obtenidos no llegan a los valores considerados como aceptables: CFI y $T L F$ igual o superior a .90, y RMSEA 
Tabla 2. Resultados del análisis factorial confirmatorio

\begin{tabular}{|c|c|c|c|c|c|c|}
\hline Modelos & $\chi^{2}$ & $\chi^{2} / g l$ & CFI & $T L I$ & RMSEA & $S R M R$ \\
\hline Modelo 1 & $\begin{array}{c}402.80 \\
(90)\end{array}$ & 4.48 & .779 & .742 & $\begin{array}{l}.104 \\
.116 \\
.127\end{array}$ & .072 \\
\hline $\begin{array}{l}\text { Modelo } 2 \\
\text { F1: } 2,3,4,5,6,7,9,12,15 \\
\text { F2: } 1,8,10,11,13,14\end{array}$ & $\begin{array}{c}355.95 \\
(89)\end{array}$ & 3.99 & .811 & .777 & $\begin{array}{l}.096 \\
.108 \\
.119\end{array}$ & .068 \\
\hline $\begin{array}{l}\text { Modelo } 3 \\
\text { F1: } 2,3,4,5,8,9 \\
\text { F2: } 1,6,7,10,11,12,13,14,15\end{array}$ & $\begin{array}{c}368.89 \\
(89)\end{array}$ & 4.14 & .802 & .766 & $\begin{array}{l}.099 \\
.110 \\
.122 \\
\end{array}$ & .073 \\
\hline $\begin{array}{l}\text { Modelo } 4 \\
\text { F1: } 2,3,4,5,6,7,9,15 \\
\text { F2: } 1,8,11,12,13,14\end{array}$ & $\begin{array}{c}274.69 \\
(76)\end{array}$ & 3.61 & .839 & .807 & $\begin{array}{l}.088 \\
.100 \\
.113\end{array}$ & .061 \\
\hline $\begin{array}{l}\text { Modelo } 5 \\
\text { F1: } 2,3,4,5,6,7,9,15 \\
\text { F2: } 1,8,11,14 \\
\text { F3: } 10,12,13\end{array}$ & $\begin{array}{c}381.12 \\
(87)\end{array}$ & 4.38 & .792 & .749 & $\begin{array}{l}.103 \\
.114 \\
.126\end{array}$ & .069 \\
\hline
\end{tabular}

Nota. CFI: comparative fit index; TLI: Tucker Lewis index; RMSEA: root mean square error of aproximation; SRMR: standardized root mean square residual.

y SRMS igual o inferior a .08 (Hu y Bentler, 1999). En suma, el modelo bifactorial de González et al. (2008) parece ajustarse mejor a los datos que el modelo unidimensional y el resto de modelos, si bien no logra un ajuste aceptable.

\section{Fiabilidad}

Se calculó la consistencia interna, la estabilidad temporal y la fiabilidad del análisis de ítems para la escala WBSI. El coeficiente de fiabilidad obtenido para la escala global fue de .88. Las correlaciones variaron entre .47 (ítem 1) y .75 (ítem 12).

El cálculo de la fiabilidad test-restest $(n=50)$, transcurrido un intervalo de 3 a 6 semanas, fue realizado mediante el coeficiente de correlación intraclase. El ICC obtenido fue bueno para el total del WBSI: 0.61 $\left(\mathrm{IC}_{95} \%=0.31-0.78\right)$.

\section{Validez convergente}

Para el análisis de la validez convergente del WBSI, fue analizada la relación de este instrumento con otras medidas que evalúan constructos teóricamente semejantes. Con este objetivo, fueron analizadas las correlaciones entre este instrumento y algunas variables psicopatológicas, concretamente obsesividad (MOCI) depresión, ansiedad y tendencia general a preocuparse de forma excesiva. De acuerdo con los criterios establecidos por Cohen (1988), el total del WBSI mostró una relación po- sitiva baja con la medida de obsesiones y compulsiones (MOCI) $(r=.18, p<.01)$, a la par que moderada con las medidas de ansiedad como rasgo (STAI-R) $(r=.36, p<$ $.01)$ y como estado (STAI-E) $(r=.34, p<.01)$. Se observó una correlación positiva y moderada entre el total del WBSI y las estrategias utilizadas para controlar pensamientos negativos en general, evaluadas con el TCQ ( $r=$ $.31, p<.01)$. Finalmente, la asociación encontrada entre el WBSI total y la tendencia para la preocupación excesiva (PSWQ) fue positiva y de grado moderado-alto $(r=$ $.47, p<.01)$.

\section{Datos normativos}

El valor medio de la puntuación total del WBSI para la muestra total fue de $47.67(D T=11.10)$, lo que muestra una moderada tendencia para la supresión de pensamientos indeseados en las personas encuestadas. Cuando se compararon los resultados en el WBSI en función del sexo de los sujetos, se observó que las mujeres $(M=$ 48.83; $D T=10.87)$ presentaron significativamente $\left(t_{(348,387)}=4.424 ; p=0,001 ; d\right.$ de Cohen $\left.=.41\right)$ una mayor tendencia para la supresión de pensamiento que los hombres $(M=44.25 ; D T=12.00)$. La magnitud del efecto fue moderada. La muestra fue dividida en tres intervalos de edad: jóvenes (18 a 30 años), adultos-jóvenes (30 a 45 años) y adultos-mayores (a partir de 45 años). No se encontraron diferencias en los tres grupos en la tendencia general a la supresión de pensamientos $(F=$ $2.591 ; p=.076$, Eta cuadrado $=.01)$. 


\section{Discusión}

La supresión de pensamiento parece ser un constructo transdiagnóstico (Belloch, 2012) que se encuentra asociado a diversas patologías tales como el trastorno obsesivo-compulsivo, el trastorno de ansiedad generalizad o la depresión. Dada la necesidad de contar con instrumentos psicométricamente válidos para poder evaluarlo, el objetivo de este estudio fue ofrecer evidencias de validez factorial y convergente de la versión portuguesa de la escala WBSI (Wegner y Zanakos, 1994).

En relación a la estructura factorial del WBSI, los resultados obtenidos no permiten aclarar cuál de las tres estructuras (unidimensional, de dos factores o de tres factores) es la más apropiada para el inventario. No obstante, los resultados del análisis factorial exploratorio se ajustaron a una estructura unidimensional tal y como se formuló en el estudio original (Wegner y Zanakos, 1994). La aparición de dos o de tres factores en otros estudios de validación del WBSI en otras lenguas (González et al., 2008; Höping y De Jong-Meyer, 2003; Ponciano et al., 2008) puede deberse al método de extracción utilizado en el análisis factorial exploratorio, el método de componentes principales, y al criterio de extracción de factores, el criterio de Kaiser. Ambos métodos tienden a sobreestimar el número de factores dado que analizan toda la varianza, común y específica, de los ítems. Por otro lado, el método de componentes principales no es considerado como el método más adecuado para la realización de un análisis factorial exploratorio (Costello y Osborne, 2005; Osborne y Fitzpatrick, 2011). El hecho de que tanto en los estudios que se ha utilizado el mismo método (ejes principales) (i.e., González et al., 2008; Palm y Strong 2007; Wegner y Zanakos, 1994), como en el presente trabajo se haya obtenido la misma estructura unidimensional apoyaría la hipótesis de la influencia del tipo de método de extracción sobre el número de factores encontrado en otros estudios (Blumberg, 2000; Höping y de Jong-Meyer, 2003; Rassin, 2003).

El objetivo principal de este estudio fue proporcionar evidencias acerca de la estructura interna, la fiabilidad y validez de la versión portuguesa del WBSI. La estructura interna e este instrumento no es del todo clara y suscita bastante controversia. De acuerdo con los resultados de los estudios realizados para su elaboración, el WBSI parecía ser una medida unifactorial, estable en el tiempo, que podría permitir, junto con otras medidas, seleccionar los sujetos más vulnerables al desarrollo de psicopatología (Wegner y Zanakos, 1994). Sin embargo, los estudios de adaptación de esta medida a otros idiomas han producido estructuras factoriales poco homogéneas. En el presente estudio, se sometieron a contraste la generalidad de las estructuras anteriormente referidas. Los resultados del análisis factorial exploratorio nos permitieron confirmar nuestra primera hipótesis, que la versión portuguesa del WBSI estaría compuesta por una única dimensión a semejanza de los resultados obtenidos por Muris et al. (1996), Palm y Strong (2007), Spinhoven y van der Does (1999) y Wegner y Zanakos (1994), pero que difiere de los encontrados por el resto de los autores.

Sin embargo, la estructura unidimensional de la escala no presenta el mejor ajuste cuando se comparan los modelos de uno, dos y tres factores mediante análisis factorial confirmatorio. Los mejores índices de ajuste se obtuvieron para el modelo bifactorial propuesto por González et al. (2008), pero tampoco alcanzó los mínimos requeridos para un buen ajuste (Byrne, 2001; Hu y Bentler, 1999). Dado que ninguno de los modelos probados mediante el análisis confirmatorio mostró un ajuste adecuado y considerando los resultados del análisis exploratorio, se puede considerar que el WBSI parece mostrar una estructura unidimensional conforme a la formulación original del cuestionario por parte de Wegner y Zanakos (1994).

Tal como ha venido siendo puesto de manifiesto por algunos autores (i.e., Muris et al.,1996; Schmidt et al., 2009; Spinhoven y van der Does, 1999), el examen atento del contenido semántico del WBSI permite identificar algunos ítems más relacionados con intrusión o incluso con estrategias de distracción que con supresión. Tanto desde el punto de vista clínico, como del de investigación, es importante poder distinguir claramente los constructos evaluados por los instrumentos. En el caso concreto del WBSI, sería necesario distinguir entre tendencia de supresión, pensamientos intrusos y estrategias de distracción. En la actualidad, existen varios instrumentos que permiten evaluar las intrusiones (i.e. The international Intrusive Thoughts Interview Schedule, RCIF, 2007) y las estrategias (i.e., TCQ, Wells y Davies, 1994). Los instrumentos que evalúan de forma inequívoca la tendencia a la supresión son, sin embargo, mucho más escasos. Para colmatar este déficit, podrían ser, desde nuestro punto de vista, eliminados del WBSI algunos de los ítems que no parecen evaluar supresión, aumentando, de esta forma, la validez de constructo de este instrumento. La dificultad estriba en la identificación de los ítems que podrían ser eliminados para que el WBSI pudiese mantener su coherencia en los diferentes idiomas. Schmidt et al. (2009) identificaron, en las diferentes versiones del WBSI que analizaron, cinco ítems que saturaban de forma consistente en el factor supresión. Sin embargo, ni en la versión española de González et al. (2000), ni en la resultante del presente trabajo se en- 
cuentra esta estructura factorial. Futuros estudios con muestras clínicas y no clínicas podrán arrojar más luz sobre la estructura interna del instrumento y la validez de constructo de los ítems.

Relativamente a la fiabilidad del WBSI, la consistencia interna obtenida fue satisfactoria y con valores semejantes a los obtenidos en los estudios donde observaron la estructura unidimensional. Igualmente, la estabilidad temporal registrada en este estudio puede ser considerada como buena, si bien ligeramente inferior a los valores obtenidos por Wegner y Zanakos (1994), Muris et al. (1996) y Höping y De Jong-Meyer (2003). No obstante, los valores obtenidos en los anteriores estudios pueden estar sobreestimados dado que su cálculo fue realizada a través del coeficiente de correlación de Pearson (Bartko, 1991).

En relación a la validez convergente, las relaciones observadas entre las puntuaciones del WBSI y las puntuaciones del MOCI, STAI, TCQ y PSWQ fueron coherentes con la literatura científica consultada. Mayores puntuaciones en variables psicopatológicas como obsesividad y ansiedad se asociaron a mayores puntuaciones en la supresión del pensamiento. En este sentido, existe consenso en considerar que aquellos individuos con mayor facilidad para suprimir pensamientos indeseados manifiestan niveles más reducidos de obsesividad (Hardy y Brewin, 2005; Smari y Hermodsdottir, 2001). Son además congruentes con los obtenidos en las investigaciones sobre el estudio de las propiedades psicométricas del WBSI anteriormente comentadas (i.e., Höping y de Jong-Meyer, 2003; Luciano et al., 2006; Muris et al., 1996; Wegner y Zanakos, 1994).

Los resultados obtenidos también apoyan que la supresión pueda ser considerada como una estrategia de control del pensamiento (Belloch, Morillo, y García-Soriano, 2007). Mayores puntuaciones en supresión se asociaron con mayores puntuaciones en las estrategias de control de pensamientos negativos.

Finalmente, los participantes del estudio mostraron una moderada tendencia para la supresión de pensamientos indeseados, siendo las mujeres las que más recurrieron a esta estrategia de control del pensamiento. Este resultado es consistente con las evidencias encontradas en la literatura sobre la validación del WBSI (Blumberg, 2000; Spinhoven y van der Does, 1999; Wegner y Zanakos, 1994).

La versión portuguesa del WBSI es un instrumento psicométricamente válido para evaluar la tendencias de supresión del pensamiento para población general. No obstante, es necesario tener en cuenta algunas limitaciones que presenta este trabajo. En primer lugar, si bien la muestra es considerablemente elevada, no se trata de una muestra representativa de la población general por- tuguesa. En segundo lugar, los datos fueron extraídos de cuestionarios aplicados sólo a participantes de la población general y no de poblaciones clínicas. Futuros estudios deben centrarse en analizar las características psicométricas del WBSI igualmente con diversas muestras clínicas. Finalmente, si bien se realizó un análisis test-restest con un periodo de entre 3 y 6 semanas, sería necesario llevar a cabo estudios longitudinales con periodos más largos y muestras mayores para verificar la estabilidad de la versión portuguesa del WBSI a lo largo del tiempo.

En síntesis, la versión portuguesa del WBSI se ajusta mejor a una estructura unidimensional y presenta niveles adecuados de fiabilidad y validez, lo que la faculta para ser utilizada en Portugal. Este instrumento permite evaluar adecuadamente los niveles de supresión de pensamiento en población no clínica y puede ser utilizada conjuntamente con otros inventarios para la evaluación psicológica.

\section{Referencias}

Bartko, J. J. (1991). Measurement and reliability: Statistical thinking considerations. Schizophrenia Bulletin, 17, 483-489.

Belloch, A. (2012). Propuestas para un enfoque transdiagnóstico de los trastornos mentales y del comportamiento: Evidencia, utilidad y limitaciones. Revista de Psicopatología y Psicología Clínica, 17, 295-311.

Belloch, A., Morillo, C., y García-Soriano, G. (2007). Obsessive themes, evaluative appraisals, and thought control strategies: Testing the autogenous-reactive model of obsessions. International Journal of Clinical and Health Psychology, 7, 5-20.

Blumberg, S. (2000). The White Bear Suppression Inventory: revisiting its factor structure. Personality and Individual Differences, 29, 934-950.

Byrne, B. M. (2010). Structural equation modeling with AMOS. Basic concepts, applications, and programming ( $2^{\mathrm{a}} \mathrm{ed}$ ed.). New York: Routledge Taylor \& Francis Group.

Cabrera-Nguyen, P. (2010). Author guidelines for reporting scale development and validation results in the Journal of the Society for Social Work and Research. Journal of the Society for Social Work and Research, 1, 99-103.

Cattell, R. B., y Vogelmann, S. (1977). A comprehensive trial of the scree and $\mathrm{KG}$ criteria for determining the number of factors. Multivariate Behavioral Research, 12, 289-325.

Cohen, J. (1988). Statistical power analysis for the behavioral sciences ( $2^{\mathrm{a}}$ ed.). Hillsdale, NJ: Erlbaum.

Costello, A., y Osborne, J. W. (2005). Best practices in exploratory factor analysis: Four recommendations for getting the most from your analysis. Practical Assessment, Research \& Evaluation, 10.

Fleiss, J. L. (1986). The design and analysis of clinical experiments. New York: Wiley.

González, M., Avero, P., Rovella, A. T., y Cubas, R. (2008). Structural validity and reliability of the Spanish version of the White Bear Suppression Inventory (WBSI) in a sample of the ge- 
neral Spanish population. Spanish Journal of Psychology, 11, 650-659.

Hambleton, R. K., Merenda, P. F., y Spielberger, C. D. (2006). Adapting educational and psychological test for cross-cultural assessment. London: Lawrence Erlbaum Associates.

Hardy, A., y Brewin, C. R. (2005). The role of thought suppression in the development of obsessions. Behavioural and Cognitive Psychotherapy, 33, 61-69.

Hodgson, R., y Rachman, S. (1977). Obsessional compulsive complaints. Behaviour Research and Therapy, 15, 398-395.

Höping, W., y de Jong-Meyer, R. (2003). Differentiating unwanted intrusive thoughts from thought suppression: what does the White Bear Suppression Inventory measure? Personality and Individual Differences, 34, 1049-1055.

Hu, L., y Bentler, P. M. (1999). Cutoff criteria for fit indexes in covariance structure analysis: Conventional criteria versus new alternatives. Structural Equation Modeling: A Multidisciplinary Journal, 6, 1-55.

Jiménez-Ros, A. (2010). Pensamientos intrusos obsesivos, valoraciones y estrategias de control en individuos no clinicos portugueses. Tesis doctoral sin publicar, Universidad de Huelva, España.

Kaiser, H. F. (1960). The application of electronic computers to factor analysis. Educational and Psychological Measurement, 20, 141-151.

Luciano, J. V., Belloch, A., Algarabel, S., Tomás, J. M., Morillo, C., y Lucero, M. (2006). Confirmatory factor analysis of the White Bear Suppression Inventory and the Thought Control Questionnaire: A comparison of alternative models. European Journal of Psychological Assessment, 22, 250-258.

Marôco, J. (2010). Análise de equações estruturais. Fundamentos teóricos, software e aplicações. Lisboa: ReporNumber, Lda.

Meyer, T. J., Miller, M. L., Metzger, R. L., y Borkovec, T. D. (1990). Development and validation of the Penn State Worry Questionnaire. Behaviour Research and Therapy, 28, 487495.

Muris, P., Merckelbach, H., y Horselenberg, R. (1996). Individual differences in thought suppression. The White Bear Suppression Inventory: Factor structure, reliability, validity and correlates. Behaviour Research and Therapy, 34, 501-513.

Muris, P., Merckelbach, H., Horselenberg, R., Sijsenaar, M., y Leeuw, I. (1997). Thought suppression in spider phobia. Behaviour Research and Therapy, 35, 769-774.

Nunnally, J. C., y Bernstein, I. J. (1995). Teoría psicométrica. Madrid: McGraw-Hill.

O'Connor, B. P. (2000). SPSS and SAS programs form determining the number of components using parallel analysis and Velicer's MAP test. Behaviour Research Methods, Instruments \& Computers, 32, 396-402.
Osborne, J. W., y Fitzpatrick, D. C. (2011). Replication analysis in exploratory factor analysis: What it is and why it makes your analysis better. Practical Assessment, Research \& Evaluation, $17,1-8$.

Palm, K. M., y Strong, D. R. (2007). Using item response theory to examine the White Bear Suppression Inventory. Personality and Individual Differences, 42, 87-98.

Ponciano, E., Daniel, F., Simões, D., Rodrigues, M. J., Medeiros, T., y Jardim, H. (2008). Validade e propriedades psicométricas do State-Trait Anxiety Inventory em estudantes universitários e do ensino secundário. Póster presentado en el $7^{\circ}$ Congresso Nacional de Psicologia da saúde, Porto.

Rassin, E. (2003). The White Bear Suppression Inventory (WBSI) focuses on failing suppression attempts. European Journal of Personality, 17, 285-298.

RCIF (2007). The International Intrusive Thoughts Interview Schedule, Versión 6. Barcelona, España.

Sandín, B., Chorot, P., Valiente, R. M., y Lostao, L. (2009). Validación española del cuestionario de preocupación PSWQ: Estructura factorial y propiedades psicométricas. Revista de Psicopatología y Psicología Clínica, 14, 107-122.

Schmidt, R., Gay, F., Courvoisier, D., Jermann, F., Ceschi, G., David, M. et al., (2009). Anatomy of the White Bear Suppression Inventory (WBSI): A review of previous findings and a new approach. Journal of Personality Assessment, 91, 323-330.

Smari, J., y Hermodsdottir, I. H. (2001). Obsessive-compulsive symptoms, white noise and intrusions of self-relevant negative thoughts in a thought suppression paradigm. Scandinavian Journal of Psychology, 42, 453-458.

Spielberger, C. D., Gorsuch, R. L., Lushene, R. E., Vagg, P. R.,y Jacobs, G. A. (1983). Manual of the State-Trait Anxiety Inventory (form Y). Palo Alto, C.A: Consulting Psychologists Press, Inc.

Spinhoven, P., y van der Does, A. J. W. (1999). Thought suppression, dissociation and psychopathology. Personality and Individual Differences, 27, 877-886.

Wegner, D. M., Schneider, D., Carter, D., y White, T. (1987). Paradoxical effects of thougth suppression. Journal of Personality and Social Psychology, 53, 5-13.

Wegner, D. M., y Zanakos, S. (1994). Chronic thought suppression. Journal of Personality, 62, 616-640.

Wenzlaff, R. M., y Wegner, D. M. (2000). Thought suppression. Annual Review of Psychology, 51, 59-91.

Wenzlaff, R. M., Wegner, D. M., y Roper, D. W. (1988). Depression and mental control: The resurgence of unwanted negative thoughts. Journal of Personality and Social Psychology, 55, 882-892.

Wells, A., y Davies, M. I. (1994). The thought control questionnaire: A measure of individual differences in the control of unwanted thoughts. Behaviour Research and Therapy, 32, 871-878. 


\section{Anexo I}

WBSI

A maioria das pessoas tem alguma vez pensamentos, ideias ou imagens inoportunas que podem ser difíceis de controlar. Gostávamos de lhe perguntar por este tipo de experiências. Por favor, com base na escala que se apresenta a seguir, faça um círculo à volta da opção que melhor descreve o seu grau de concordância com cada uma delas. Não existem respostas certas ou erradas, responda apenas com sinceridade. Certifique-se que respondeu a todas as questões.

\begin{tabular}{|c|c|c|c|c|}
\hline 1 & 2 & 3 & 4 & 5 \\
\hline $\begin{array}{c}\text { Completamente em } \\
\text { desacordo }\end{array}$ & Bastante em desacordo & $\begin{array}{c}\text { Nem de acordo, nem em } \\
\text { desacordo }\end{array}$ & Bastante de acordo & $\begin{array}{c}\text { Completamente de } \\
\text { acordo }\end{array}$ \\
\hline
\end{tabular}

\begin{tabular}{|c|c|c|c|c|c|}
\hline 1. Há coisas nas que prefiro não pensar & 1 & 2 & 3 & 4 & 5 \\
\hline 2. As vezes pergunto-me porque tenho determinados pensamentos & 1 & 2 & 3 & 4 & 5 \\
\hline 3. Tenho pensamentos que não posso parar & 1 & 2 & 3 & 4 & 5 \\
\hline 4. Há imagens que aparecem na minha mente e que não posso apagar & 1 & 2 & 3 & 4 & 5 \\
\hline 5. Os meus pensamentos frequentemente giram em torno de uma só ideia & 1 & 2 & 3 & 4 & 5 \\
\hline 6. Desejava poder deixar de pensar em certas coisas & 1 & 2 & 3 & 4 & 5 \\
\hline 7. Às vezes a minha mente anda tão depressa que desejaria poder para-la & 1 & 2 & 3 & 4 & 5 \\
\hline 8. Às vezes trato de afastar os problemas da minha mente & 1 & 2 & 3 & 4 & 5 \\
\hline 9. Há pensamentos que ficam a rondar a minha cabeça & 1 & 2 & 3 & 4 & 5 \\
\hline 10. Às vezes mantenho-me ocupado para não deixar que alguns pensamentos se infiltrem na minha mente & 1 & 2 & 3 & 4 & 5 \\
\hline 11. Há coisas sobre as que tento não pensar & 1 & 2 & 3 & 4 & 5 \\
\hline 12. Às vezes desejaria realmente poder parar o meu pensamento & 1 & 2 & 3 & 4 & 5 \\
\hline 13. Com frequência faço coisas para distrair-me dos meus pensamentos & 1 & 2 & 3 & 4 & 5 \\
\hline 14. Com frequência tenho pensamentos que tento evitar & 1 & 2 & 3 & 4 & 5 \\
\hline 15. Tenho muitos pensamentos que não conto a ninguém & 1 & 2 & 3 & 4 & 5 \\
\hline
\end{tabular}

